\title{
Maternal uniparental disomy of chromosome 20: a novel imprinting disorder of growth failure
}

\author{
Surabhi Mulchandani, MS1, Elizabeth J. Bhoj, MD, PhD², Minjie Luo, PhD', \\ Nina Powell-Hamilton, $\mathrm{MD}^{3}$, Kim Jenny, $\mathrm{MS}^{3}$, Karen W. Gripp, MD³, Miriam Elbracht, MD, \\ Thomas Eggermann, PhD ${ }^{4}$, Claire L.S. Turner, MD, I. Karen Temple, MD ${ }^{6,7}$, Deborah J.G. Mackay, PhD, \\ Holly Dubbs, MS², David A. Stevenson, $\mathrm{MD}^{8}$, Leah Slattery, MS², Elaine H. Zackai, MD², \\ Nancy B. Spinner, PhD', lan D. Krantz, MD², and Laura K. Conlin, PhD¹
}

\begin{abstract}
Purpose: Maternal uniparental disomy of chromosome 20 (UPD(20) mat) has been reported in only four patients, three of whom also had mosaicism for complete or partial trisomy of chromosome 20 . We sought to evaluate the clinical significance of isolated $\operatorname{UPD}(20)$ mat in eight individuals.
\end{abstract}

Methods: We evaluated phenotypic and genomic findings of a series of eight new patients with UPD(20)mat.

Results: All eight individuals with UPD(20)mat had intrauterine growth restriction, short stature, and prominent feeding difficulties with failure to thrive. As a common feature, they often required gastric tube feeds. Genomic data in most patients are indicative of UPD as a result of trisomy rescue after meiosis II nondisjunction.
Conclusion: We describe the first natural history of the disorder and the results of therapeutic interventions, including the frequent requirement of direct gastric feedings only during the first few years of life, and propose that growth hormone supplementation is probably safe and effective for this condition. We suggest that UPD(20)mat can be regarded as a new imprinting disorder and its identification requires specialized molecular testing, which should be performed in patients with early-onset idiopathic isolated growth failure.

Genet Med advance online publication 6 August 2015

Key Words: failure to thrive; feeding difficulties; imprinting disorder; maternal uniparental disomy; short stature

\section{INTRODUCTION}

Uniparental disomy (UPD) is defined as the presence of a whole or parts of a chromosome pair originating from only one parent. Heterodisomy is the inheritance of both of the nonidentical homologous chromosomes, and isodisomy is the inheritance of two identical copies of one chromosome. Due to recombination, both heterodisomy and isodisomy can be observed in UPD. Although patients with UPD can be euploid, there can still be phenotypic consequences from altered gene expression due to imprinted genes, from residual aneuploidy, or in isodisomy from uncovering mutations associated with a recessive disorder.

Currently, eight human imprinting syndromes have been reported with a diverse range of phenotypes, although most involve aberrant growth (for review: http://upd-tl.com/upd. html). Chromosomes 6, 7, 11, 14, 15, and 20 harbor imprinted genes associated with well-described syndromes, and the phenotypes from maternal and paternal UPD of the same chromosome can be strikingly different. ${ }^{1}$ For example, paternal UPD 14 leads to variable developmental delay, dysmorphic facial features, skeletal abnormalities, and joint contractures, but maternal UPD 14 patients (Temple Syndrome) demonstrate intrauterine growth failure (IUGR), growth delay, central hypotonia, developmental delay, and early onset of puberty.,

There have been only a few patients reported with UPD of chromosome 20 (ref. 4). UPD(20)pat results in pseudohypoparathyroidism type 1b (OMIM 603233), which is characterized by resistance to parathyroid hormone in kidneys and presents as hypocalcemia, hyperphosphatemia, and abnormally high parathyroid hormone levels. There are only a few patients with UPD(20)mat who have been described in the literature. Complicating their characterization, most of these patients with UPD(20)mat had other related structural alterations resulting in partial or complete trisomy for chromosome 20. The first reported patient with UPD(20)mat had a mosaic karyotype with $46, \mathrm{XY}$ and $47, \mathrm{XY}$, +mar, with the marker chromosome comprising the centromere and pericentromeric segments of chromosome $20 .{ }^{4}$ A second patient was diagnosed prenatally with mosaic trisomy 20 and was nonmosaic for UPD(20)mat in blood postnatally. ${ }^{5}$ A third patient with mosaic trisomy 20 had trisomy in $98 \%$ of amniotic fluid, $100 \%$

The first two authors contributed equally to this work.

${ }^{1}$ Division of Genomic Diagnostics, Children's Hospital of Philadelphia, Philadelphia, Pennsylvania, USA; ${ }^{2}$ Division of Clinical Genetics, Children's Hospital of Philadelphia, Philadelphia, Pennsylvania, USA; ${ }^{3}$ Division of Medical Genetics, Alfred I. duPont Hospital for Children, Wilmington, Delaware, USA; ${ }^{4}$ Institute of Human Genetics, University Hospital, RWTH Aachen, Aachen, Germany; ${ }^{5}$ Peninsula Clinical Genetics Service, Royal Devon and Exeter Hospital, Exeter, UK; ${ }^{6}$ Wessex Clinical Genetics Service, University Hospital Southampton, Southampton, UK; ${ }^{7}$ Human Development and Health, Faculty of Medicine, University of Southampton, Southampton, UK; ${ }^{8}$ Division of Medical Genetics, Stanford University, Stanford, California, USA. Correspondence: Elizabeth J. Bhoj (bhoje@email.chop.edu) 
of placenta and urine sediment, and 10\% of peripheral blood. ${ }^{6}$ The presence of trisomy in these individuals complicates the clinical implication of UPD(20)mat. A fourth patient was identified by screening 51 patients with IUGR and postnatal growth delay; no trisomic cells were identified, making him the only reported case of isolated UPD(20)mat. ${ }^{7}$ Additionally, one case of UPD(20)mat with nonmosaic trisomy 20p is included in the UPD database (http://upd-tl.com/upd.html). This was a 16-month-old male with short stature, dysmorphic features, right inguinal hernia, hypospadias, mild cardiac defect, and feeding difficulties that required gastric feeding. The method of UPD detection is unclear for this patient; therefore, this case is not used for further comparison.

Eight new individuals with UPD(20)mat without evidence of trisomy are described in this report. All individuals share the phenotype of IUGR and "idiopathic" failure to thrive (FTT) severe enough that six out of eight patients required chronic gastric tube feedings. Beyond the growth phenotype, there are no additional shared features. The probands are not overtly dysmorphic and have no major congenital abnormalities. Three of eight patients were reportedly affected with fifth-finger clinodactyly, which is higher than in the general population. They do not demonstrate major developmental delay, and all are currently in mainstream education working at grade level. Therefore we suggest that UPD(20)mat can be regarded as a novel imprinting disorder of IUGR, extreme feeding difficulties from birth, and short stature.

\section{METHODS}

This study was deemed exempt by the official Institutional Review Board at the Children's Hospital of Philadelphia and the other contributing institutions. Informed consent was obtained, including written consent for all published photographs. Clinical testing using the single-nucleotide polymorphisms (SNP) chromosomal microarray platforms Illumina $610 \mathrm{k}$, OMNI $1 \mathrm{M}$, and $850 \mathrm{k}$ arrays and Affymetrix Cytoscan identified UPD(20)mat in patients 1 to 5 and 8. In general, UPD was suspected in samples with homozygosity limited to only one chromosome, particularly when greater than $10 \mathrm{Mb}$ in size or terminally located. ${ }^{1,8}$ Confirmation of UPD was performed by analyzing several thousand informative parental genotypes across the entire chromosome 20. Patient 6 was identified by methylation-specific multiplex ligation-dependent probe amplification (MS MLPA) using a GNAS-specific assay (ME-031-B; MRC Holland, Amsterdam, The Netherlands). UPD(20)mat was then confirmed by typing of chromosome 20 -specific microsatellite markers and SNP array analysis (Affymetrix 6.0 SNP array, Santa Clara, CA). Patient 7 was identified through recruitment to the Imprinting Disorders Finding Out Why Study (IDFOW: Southampton and South West Hampshire Ethics approval 07/H0502/85) and investigated by methylation-specific polymerase chain reaction of multiple imprinted loci, including those on chromosome 20. Results were confirmed by analysis of chromosome 20 -specific microsatellites. ${ }^{9}$

\section{RESULTS}

\section{Patient 1}

Patient 1 was a 9-year-old girl with short stature and feeding difficulties from birth (Figure 1). She was born to a 39-yearold mother after a spontaneous pregnancy at 38 weeks of gestation with a birth weight of $2,200 \mathrm{~g}(<2$ nd percentile); there were no complications during the pregnancy or delivery. At age 3 months she was diagnosed with FTT attributed to reflux. Despite aggressive medical management of the reflux she required nasogastric tube feeding from age 3 months to 3 years. She was first evaluated by medical genetics at 8 months for isolated FTT. Chromosome analysis, subtelomere analysis, fluorescent in situ hybridization testing for 22q11.2 deletion, and a comprehensive metabolic panel were all normal. The patient was started on growth hormones $(\mathrm{GH})$ at age 8 years for idiopathic short stature after GH stimulation testing and SHOX gene sequencing were normal. She also had a bone age that was consistent with chronologic age and showed no signs of puberty. She grew 4 inches during the first year on GH. However, during this time she was diagnosed with scoliosis and wore a back brace for 16 hours per day; she did not have any central hypotonia or other predisposing factors for scoliosis. Academically, she worked at grade level in school and did not require extra help in the classroom. At age 9 years she had an unremarkable physical examination with no dysmorphic features; height was 121 centimeters $(\mathrm{cm})$ (5th percentile) and weight was $23 \mathrm{~kg}$ (5th percentile). Her family history was unremarkable. A genome-wide SNP array identified homozygosity for the entire chromosome 20. Parental testing confirmed maternal origin of UPD.

\section{Patient 2}

Patient 2 was born to a 38-year-old mother at full term weighing 2,600 g (2nd percentile). She first came to medical attention during the newborn period because of poor feeding, which was thought to be due to "sensory issues." She required nasogastric tube feedings from 9 months to 2 years, but she still had poor growth through that time despite adequate caloric intake. There are no reported developmental issues, and she was performing without extra help at grade level. At her last examination at age 8 , her height was $110.9 \mathrm{~cm}$ (<3rd percentile; 50 th percentile for 5.5 years), weight was $15.9 \mathrm{~kg}$ ( $<3 \mathrm{rd}$ percentile; 50th percentile for 4 years), and head circumference was $49.5 \mathrm{~cm}$ (3rd percentile). She had mildly low-set ears with thickened helices bilaterally and a narrow palate. She also had multiple lightly pigmented spots on her back, chest, abdomen, extremities, and head with a few darker macules not consistent with classic café-au-lait spots due to their color and borders. She did not have a bone age test performed and showed no signs of puberty. Her family history was unremarkable. The proband had negative testing for LEOPARD syndrome and neurofibromatosis. A genome-wide SNP array identified homozygosity for the entire chromosome 20; parental samples have been requested to confirm parent of origin. 


\section{Patient 3}

Patient 3 was a boy born by vaginal delivery to a 43 -year-old mother after a spontaneous pregnancy at 38 weeks of gestation with a birth weight of $2,400 \mathrm{~g}$ ( $3 \mathrm{rd}$ percentile). There were no pregnancy complications and an amniocentesis performed for advanced maternal age reported normal 46XY. The patient had problems gaining weight in the first months of life; at 19 months he weighed $8.6 \mathrm{~kg}(<5$ th percentile, 50 th percentile for 8 months), and at the most recent examination at age 4 he had a height of $78 \mathrm{~cm}(<5$ th percentile, 50th percentile for 14 months). Due to FTT from poor oral intake, he required nasogastric tube feeds from age 22 months to 4 years. He also had a tonsillectomy to improve potential dysphagia affecting his ability to consume enough calories, but it did not improve his intake significantly. His developmental milestones were all normal. Family history was significant only for his mother having a miscarriage with a trisomy 18 karyotype. On his last examination at age 4 years his height was $80.5 \mathrm{~cm}$ ( 5 th percentile), his weight was $9.32 \mathrm{~kg}$ ( $<5$ th percentile, 50 th percentile for 12 months), and he had a head circumference of $47.75 \mathrm{~cm}$ (25th percentile). He had epicanthic folds and one lightly pigmented spot, but no other abnormalities. A genome-wide SNP array identified an interstitial $28.5 \mathrm{Mb}$ region of homozygosity from chromosome 20p12.1to q13.13 spanning the centromere. Parental testing confirmed maternal origin of the UPD.

\section{Patient 4}

Patient 4 was the male product of intrauterine insemination secon dary to lack of spontaneous conception after 2 years. The pregnancy was complicated by IUGR, first noticed at 32 weeks of gestational age, and decreased fetal movement toward the end of the pregnancy. He was born to his 43-year-old mother at 39 weeks of gestation with a birth weight of $1,960 \mathrm{~g}(<3 \mathrm{rd}$ percentile, 50th percentile for 32 weeks of gestation) and a length of $43 \mathrm{~cm}$ ( $<3 \mathrm{rd}$ percentile, 50th percentile for 32 weeks of gestation). He was admitted to the neonatal intensive care unit for poor weight gain and temperature instability. Owing to "fatigue when eating" and "texture issues" he was placed on nasogastric tube feeds from age 1 year until 4 years. Additional medical history was notable for a horseshoe kidney, a ventricular septal defect that closed spontaneously, mild asthma, egg allergies, and late eruption of his molar teeth. He also had generalized hypotonia with gross motor delays that placed him approximately 6 months behind on his milestones globally; he had normal brain magnetic resonance imaging results at age 6 weeks. He did well in a regular classroom setting without additional help. At his last examination at age 5 years he weighed $21 \mathrm{~kg}$ (75th percentile) and his height was $108 \mathrm{~cm}$ (10th percentile). There were no dysmorphic facial features, but he had a bifid left second toe. A genome-wide SNP array identified an interstitial $40.80 \mathrm{Mb}$ region of homozygosity affecting most of chromosome 20 (20p12.3q13.13), spanning the centromere (Figure 2d). Parental testing confirmed maternal origin of the UPD.

\section{Patient 5}

Patient 5 was a boy born to a 37-year-old mother via cesarean delivery at 37 weeks of gestation for maternal hypertension. The pregnancy was complicated by oligohydramnios late in the pregnancy and breech presentation. The patient had IUGR with a birth weight of $2,050 \mathrm{~g}$ ( $<5$ th percentile, 50th percentile for 34 weeks of gestation), length of $46.5 \mathrm{~cm}$ (5th percentile), and a head circumference of $31.5 \mathrm{~cm}$ ( $<5$ th percentile, 50 th percentile for 33.5 weeks). He remained in the neonatal intensive care unit for 7 days for feeding difficulty and hypoglycemia. His feeding issues persisted despite medical management, and he was found to be hypotonic at 4 months. A neuromuscular workup including muscle ultrasound was unable to provide a diagnosis for his hypotonia. At 8 months he began feeds via nasogastric tube, which was converted to a percutaneous gastric tube at 18 months. He continued to have slow growth with a slightly delayed bone age; despite normal levels of $\mathrm{GH}$ he was started on supplemental $\mathrm{GH}$ therapy at 5 years, which resulted in moderate growth acceleration. By age 6 years he was able to support his growth without gastric feeds and his gastrostomy tube was removed. He was in a normal classroom at grade level and doing well. His family history was remarkable for Stickler syndrome in a paternal uncle, paternal aunt, and paternal cousin, but not in his father. On most recent examination at age 6 the patient had bilateral fifth-finger brachyclinodactyly and one irregular patch of increased pigmentation on his left thigh but was otherwise nondysmorphic. His height was $104 \mathrm{~cm}$ (<3rd percentile, 50th percentile for 4.5

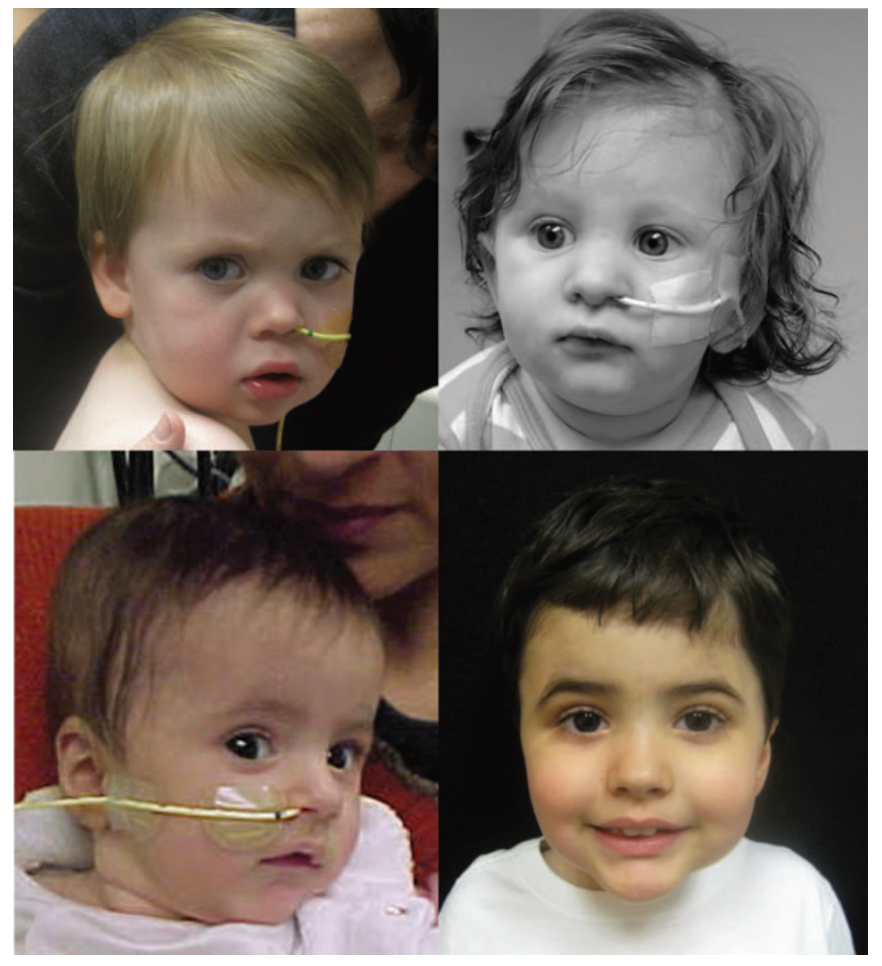

Figure 1 Patients with maternal uniparental disomy of chromosome 20; there are no overt facial dysmorphisms. Upper panel left to right: Patient 1 at 11 months and patient 2 at 14 months. Lower panel left to right: Patient 4 at 12 months and again at 4 years. 

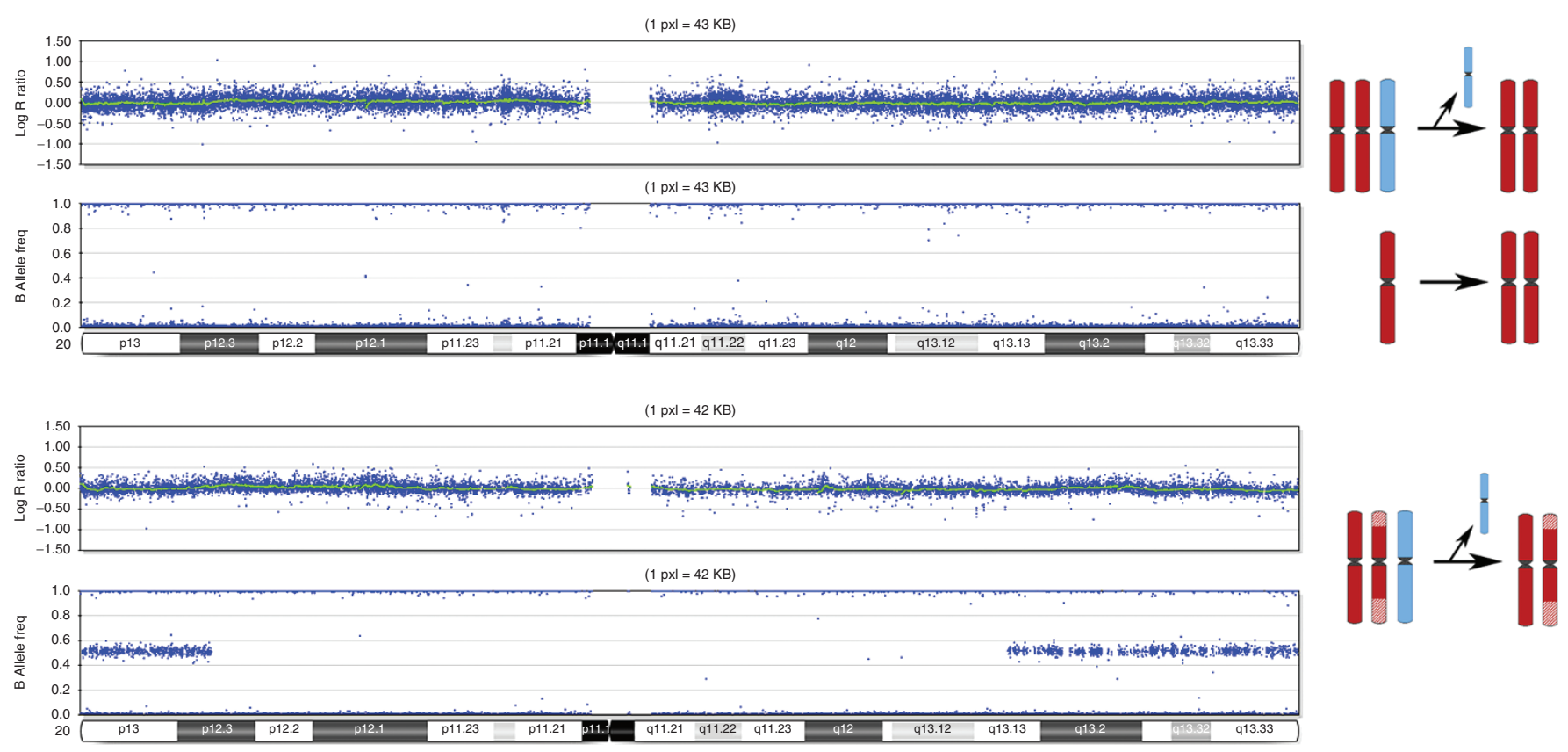

Figure 2 Chromosomal SNP array results and plausible mechanism for formation. (a) Homozygosity over entire chromosome 20. (b) Homozygosity spanning the centromere. SNP, single-nucleotide polymorphism.

years), he weighed $14.4 \mathrm{~kg}$ ( $<3 \mathrm{rd}$ percentile, 50th percentile for 3.5 years), and head circumference was $49.25 \mathrm{~cm}$ (25th percentile). He was originally given a clinical diagnosis of Silver-Russell Syndrome (SRS) before he was found to have UPD(20)mat. A SNP chromosomal array detected two regions of homozygosity, a $6 \mathrm{Mb}$ region on the $\mathrm{p}$ terminus, and a $20 \mathrm{Mb}$ interstitial region from 20p11.21 to q13.12 spanning the centromere. Parental testing confirmed maternal origin of the UPD.

\section{Patient 6}

Patient 6 was a 12 -year-old boy. After an uneventful pregnancy, he was born spontaneously to a 38-year-old mother in the 38th week of gestation. He was small for gestational age (SGA) at a length of $47 \mathrm{~cm}$ (3rd percentile) and a weight of 2,240 $(<1 \mathrm{st}$ percentile, 50th percentile for 35 weeks). He demonstrated feeding difficulties with poor sucking, but breastfeeding was ultimately successful and he was also supplemented with a high-calorie diet. His growth was slow, between the first and third percentile, which was below his expected genetic potential height. At age 2 years the proband had mild muscular hypotonia with hyperlordosis, but no further dysmorphic signs, and psychomotor development was normal. Insulin growth factor-1 (IGF-1) and insulin growth factor binding protein 3 levels were initially within the normal range, but they decreased in childhood and partial GH deficiency was diagnosed by stimulation tests at the age of nearly 5 years. Since that time he had continuously used GH therapy, which led to catch-up growth to a height between the 75th and 90th percentile. He has not developed further medical problems and is in a regular classroom setting. MS MLPA using a GNAS-specific assay indicated UPD(20)mat; heterodisomy UPD(20)mat was then confirmed by microsatellite typing and SNP array analysis.

\section{Patient 7}

Patient 7 was a 9-year-old girl. She was born to a 42 -year-old mother via cesarean delivery at 39 weeks of gestation after indications for breech presentation and low amniotic fluid index. Birth weight was $2,400 \mathrm{~g}(<1$ st percentile, 50 th percentile for 35 weeks). There were early feeding problems, but nasogastric feeding was not initiated. During early childhood, she continued to demonstrate worsening proportionate symmetric FTT. At presentation at 3.75 years, her initial investigations included the following: bone age delayed by more than 1 year; normal female karyotype; normal thyroid function; negative sweat test results; no anti-tissue transglutaminase antibodies; and low normal IGF-1 levels. GH response to an insulin tolerance test at 6 years was suboptimal and brain magnetic resonance imaging results showed a normal pituitary. She has shown a good response to GH treatment, which commenced at age 7 years. On examination at age 6.5 years, her height and weight were near the 1st percentile and her head circumference was $<1$ st percentile. She had a high-pitched voice and a triangular face with posteriorly angulated ears. There was bilateral fifth-finger clinodactyly and mild syndactyly of the second and third toes. There was no skeletal asymmetry or caféau-lait patches. Her palate was intact and there were no other unusual findings on clinical examination. At age 9.3 years, her height was $119.4 \mathrm{~cm}(<2$ nd percentile), weight was $25 \mathrm{~kg}$ ( $25 \mathrm{th}$ percentile), and head circumference was $49.7 \mathrm{~cm}(<1 \mathrm{st}$ percentile). Early development was entirely normal, and there were no difficulties in meeting educational targets. Family history was noncontributory. Given the phenotypic overlap with SRS, tests for UPD7(mat) and methylation abnormalities were performed, which were normal. Methylation-sensitive polymerase chain reaction investigations at a number of additional differentially methylated regions, including three loci on chromosome 


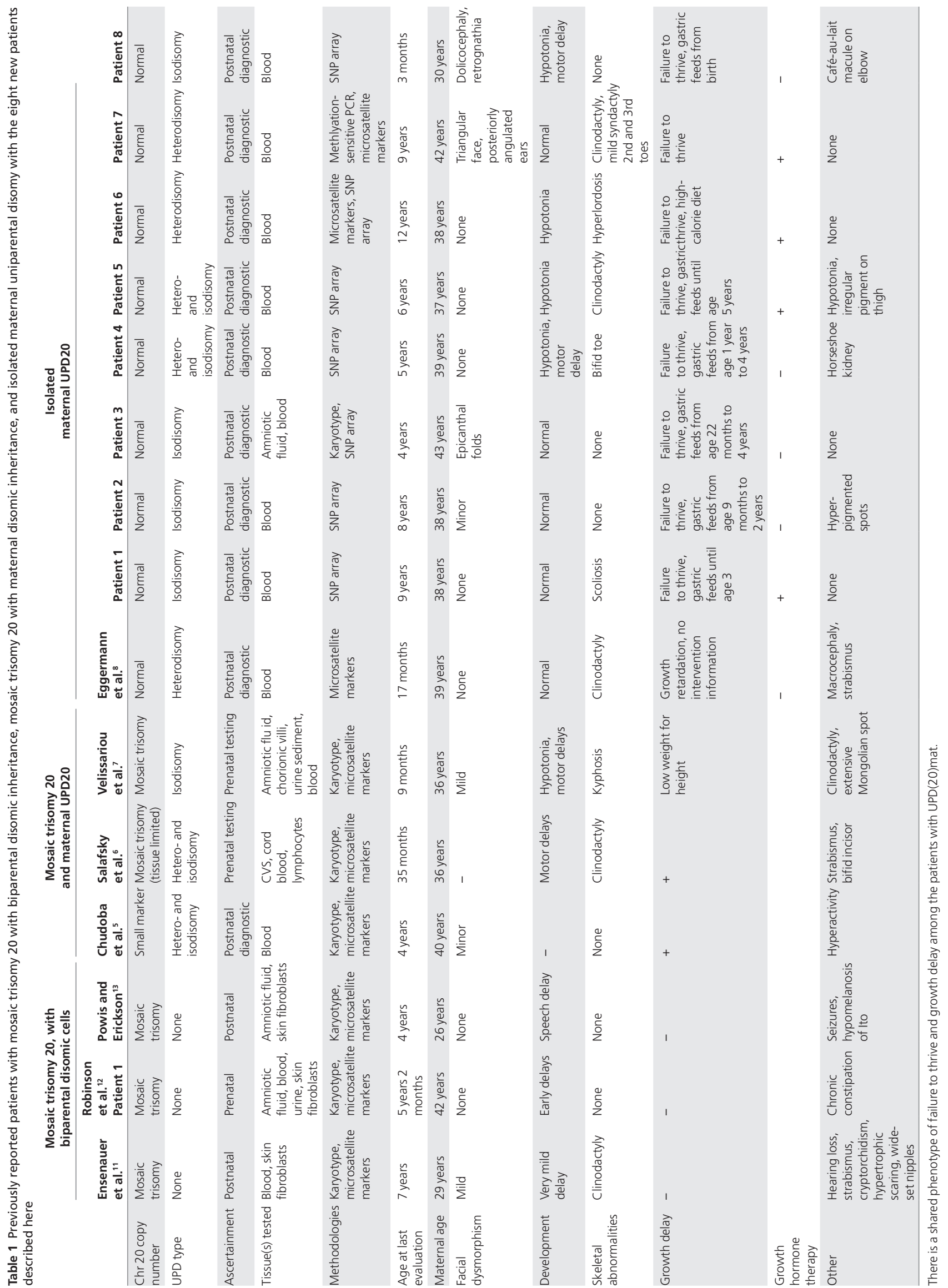


20 (NNAT, L3MBTL1, and GNAS/NESP/NESPAS), suggested UPD(20)mat. Subsequent microsatellite analyses with parental samples supported this diagnosis. In situ hybridization studies performed on uncultured buccal cells showed no evidence of trisomy 20 mosaicism.

\section{Patient 8}

Patient 8 was a 3 -month-old girl born at 36 weeks via vaginal delivery to a 30-year-old mother and 36-year-old father; prenatal ultrasound showed IUGR. Birth weight was $1.814 \mathrm{~kg}(<2 \mathrm{nd}$ percentile) and birth length was $45.7 \mathrm{~cm}(<2$ nd percentile). After birth she had significant hypotonia and feeding problems. She required gavage feedings and remained in the hospital for 4.5 weeks because of feeding problems. Magnetic resonance imaging of the brain was normal. She had hyperbilirubinemia that required phototherapy. A nasogastric feeding tube was still required at the time of discharge from the hospital. She was reevaluated at 3 months of age, and she continued to have a weak suck and hypotonia. Her height was $57 \mathrm{~cm}$ (5th percentile), weight was $3.6 \mathrm{~kg}(<2 \mathrm{nd}$ percentile), and head circumference was $36 \mathrm{~cm}(<2$ nd percentile). She was primarily fed via nasogastric tube. She had developmental delay of motor milestones but was smiling interactively. On physical examination she was relatively nondysmorphic, but minor anomalies included dolicocephaly, retrognathia, upslanting palpebral fissures, and one café au lait macule on the left elbow. There was no family history of similar problems and parents reported no consanguinity. Methylation studies for Prader-Willi syndrome were normal. An oligonucleotide SNP array was performed by Quest Diagnostics and showed $\operatorname{arr}[\mathrm{hg} 19] 20 \mathrm{p} 12.2 \mathrm{q} 13.12(11,270,110-46,395,807) \times$ $2 \mathrm{hmz}$ representing one segment of allelic homozygosity spanning the centromere at $20 \mathrm{p} 12.2 \mathrm{q} 13.12$ that was approximately $35.1 \mathrm{Mb}$. In conjunction with parental samples, molecular analysis of 11 chromosome polymorphic markers confirmed UPD(20) mat.

\section{DISCUSSION}

We present clinical and molecular evidence for a novel imprinting disorder caused by maternal uniparental disomy of chromosome 20 (UPD(20)mat syndrome) based on studies of eight new patients. This disorder is characterized by mild prenatal growth restriction, severe short stature with proportional head circumference, and profound feeding difficulty. Four previously reported patients and an additional patient from the UPD database with UPD(20)mat demonstrated this pattern of growth delay and failure to thrive; however, their diagnoses were complicated by mosaic trisomy 20 (Table 1 ). It is possible that residual undetectable trisomy mosaicism contributes to the phenotype of the patients presented here; however, the striking similarities in the phenotype implicate UPD as the causative factor. Additionally, growth delay and failure to thrive are not reported in six patients with mosaic trisomy 20 where the disomic cell line had biparental inheritance, providing additional evidence that $\mathrm{UPD}(20)$ mat is necessary for this phenotype (Table 1) ${ }^{11-13}$ Furthermore, there are no reports about UPD(20) mat carriers with a normal clinical outcome. Taken together, these findings suggest that $\mathrm{UPD}(20)$ mat leads to growth restriction, early feeding difficulties, and severe postnatal growth delay. In our series there is no evidence for significant developmental delay, because all older individuals are doing well in an age-appropriate classroom setting, although four of the eight patients have a history of hypotonia. Four of the patients in this series responded to GH treatment. This included two patients with demonstrable GH deficiency, which was also reported previously in a single case. ${ }^{8}$ Additional variable features found in previously published individual cases may be the result of the chromosomal abnormalities that lead to the formation of the UPD(20)mat, such as mosaic trisomy 20 , or homozygosity for pathogenic variants in disease-associated genes.

Although some individuals presented with additional findings, the striking phenotypic similarity is the unexplained feeding difficulty manifesting in early infancy. Generally, the infants did not show a normal hunger drive, would not wake to eat, and would not spontaneously cry to be fed. Despite extensive testing in most patients, no anatomic or physiologic etiology could be found in any of the cases. Five out of seven older patients depended on direct gastric feeds in infancy until late preschool/ early elementary school. They gained weight on feeds but may have had an increased energy requirement because their improvement was not as great as would be expected for the amount of calories consumed. At the time of last evaluation, they still displayed decreased hunger, generally eating small meals with parental encouragement.

Interestingly, a reported patient with isolated paternal UPD of $20 \mathrm{q}$ (UPD (20q)pat) was found to have parathyroid hormone resistance but was also more than 3 SDs above normal height and weight at 6 years. ${ }^{14}$ The most promising causative locus in the 20q region is GNAS, which is known to be imprinted and a cause of human disease. The GNAS locus is complex, with multiple promoters, differentially methylated regions, and parent-of-origin specific expression limited to certain tissues. Mutations in GNAS are associated with a number of diseases, including Albright's hereditary osteodystrophy and pseudohypoparathyroidism when they occur on the paternal allele and pseudopseudohypoparathyroidism on the maternal allele. Abnormalities of the paternal imprint at the GNAS region has been implicated in obesity. ${ }^{15}$ Mouse models have shown that loss of paternal alleles leads to decreased adipose tissue, a higher metabolic rate, and poor suckling. ${ }^{16-18}$ These findings suggest that GNAS plays a significant role in feeding, growth, and energy metabolism. Further studies on the GNAS expression in our patients are underway to explore its role in the UPD(20) mat phenotype. In addition, there are other potentially important imprinted loci on chromosome 20, such as L3MBTL1 and NNAT, which may also be implicated. This work may lead to insights into the basic science of hunger and metabolism control, and even pharmacologic manipulation of these areas.

Both patients 5 and 7 were investigated as possibly having SRS; there are many similarities with this phenotype, including low birth weight, FTT, postnatal growth restriction, severe feeding 
difficulties, hyperpigmented skin regions, and clinodactyly. These patients differ from SRS because there is no asymmetry, no described prominence of the forehead, and no relative macrocephaly. However, more cases need to be reported to make a clear distinction between this phenotype and that of SRS. There is also overlap with UPD(14)mat or Temple syndrome, which demonstrates growth restriction and hypotonia.

Advanced maternal age is associated with an increased risk of nondisjunction resulting in aneuploidies; most notably from meiosis I errors, but also meiosis II errors as well. ${ }^{19}$ Of note is the observation in this study that the mean maternal age is 38 years, which provides some evidence that UPD20 may be secondary to aneuploidy rescue (Supplementary Table S1 online). All five patients with high-resolution SNP array testing had genotyping patterns suggesting a meiosis II error or postzygotic mitotic error resulting in maternal UPD. Patients 1 and 2 had homozygosity over the entire chromosome (Figure 2a), and patients 3, 4, 5, and 8 had homozygosity limited to the centromeric region (Figure $2 \mathrm{~b}$ ). This pattern is consistent with trisomy rescue after meiosis II error with evidence of crossing over. The presence of complete heterodisomy would not be readily detected using SNP array technology, unless parental samples are tested up front. Therefore, it is important to perform trio testing by SNP chromosomal microarray or by performing short tandem repeat typing over multiple loci on the chromosome.

\section{Conclusion}

Here we describe UPD(20)mat as an important and novel cause of growth failure and FTT, and suggest that it may be defined as a new imprinting disorder. This diagnosis should be considered in patients with these clinical features, and $\operatorname{UPD}(20)$ mat testing should be included in the diagnostic workup. These patients each endured a lengthy diagnostic odyssey, including surgery, causing significant stress for the family and increased medical costs. Therefore, in any child with early-onset idiopathic severe feeding difficulty, UPD(20)mat should be considered. Patients who present with SRS or Temple syndrome, but exclusion of their known molecular disturbances, are strong candidates for $\operatorname{UPD}(20)$ mat because there appears to be significant phenotypic overlap. Supporting signs would include IUGR, hypotonia, fifth-finger clinodactyly, and mild abnormalities of skin pigmentation. It is difficult to estimate the prevalence of $\operatorname{UPD}(20)$ mat in patients with severe feeding difficulties, because the majority of these patients do not undergo testing that would identify this condition. In addition, it appears that GH therapy should be considered in patients with UPD $(20)$ mat, because all four patients in our series who have received it have experienced linear growth acceleration while using it. We believe that we will see increased diagnosis and treatment of UPD(20)mat as the condition becomes more recognized across multiple pediatric specialties.

\section{ACKNOWLEDGMENTS}

Funding for the evaluation of one patient was through the Newlife Foundation for Disabled Children, Wessex Comprehensive Research Network, National Institute for Health Research, and Medical Research Council. No funding for the remaining parts of the study was secured. M.E., T.E., I.K.T., and D.J.G.M. are members of the European network of congenital imprinting disorders (EUCID.net), supported by the European Cooperation in Science and Technology (COST: Action BM1208).

\section{DISCLOSURE}

The authors declare no conflict of interest.

\section{REFERENCES}

1. Kearney HM, Kearney JB, Conlin LK. Diagnostic implications of excessive homozygosity detected by SNP-based microarrays: consanguinity, uniparental disomy, and recessive single-gene mutations. Clin Lab Med 2011;31: 595-613, ix.

2. Sutton VR, Shaffer LG. Search for imprinted regions on chromosome 14: comparison of maternal and paternal UPD cases with cases of chromosome 14 deletion. Am J Med Genet 2000;93:381-387.

3. Ioannides Y, Lokulo-Sodipe K, Mackay DJ, Davies JH, Temple IK. Temple syndrome: improving the recognition of an underdiagnosed chromosome 14 imprinting disorder: an analysis of 51 published cases. J Med Genet 2014;51:495-501.

4. Venditti CP, Hunt P, Donnenfeld A, Zackai E, Spinner NB. Mosaic paternal uniparental (iso)disomy for chromosome 20 associated with multiple anomalies. Am J Med Genet A 2004;124A:294-279.

5. Chudoba I, Franke $Y$, Senger $G$, et al. Maternal UPD 20 in a hyperactive child with severe growth retardation. Eur J Hum Genet 1999;7:533-540.

6. Salafsky IS, MacGregor SN, Claussen U, von Eggeling F. Maternal UPD 20 in an infant from a pregnancy with mosaic trisomy 20. Prenat Diagn 2001;21: 860-863.

7. Velissariou V, Antoniadi T, Gyftodimou J, et al. Maternal uniparental isodisomy 20 in a foetus with trisomy 20 mosaicism: clinical, cytogenetic and molecular analysis. Eur J Hum Genet 2002;10:694-698.

8. Eggermann T, Mergenthaler S, Eggermann K, et al. Identification of interstitial maternal uniparental disomy (UPD) (14) and complete maternal UPD(20) in a cohort of growth retarded patients. J Med Genet 2001;38:86-89.

9. Papenhausen P, Schwartz S, Risheg $H$, et al. UPD detection using homozygosity profiling with a SNP genotyping microarray. Am J Med Genet $A$ 2011;155A:757-768.

10. Poole RL, Docherty LE, Al Sayegh A, et al.; International Clinical Imprinting Consortium. Targeted methylation testing of a patient cohort broadens the epigenetic and clinical description of imprinting disorders. Am J Med Genet A 2013:161A:2174-2182.

11. Ensenauer RE, Shaughnessy WJ, Jalal SM, Dawson DB, Courteau LK, Ellison JW. Trisomy 20 mosaicism caused by a maternal meiosis II error is associated with normal intellect but multiple congenital anomalies. Am J Med Genet A 2005;134A:202-206.

12. Robinson WP, McGillivray B, Lewis ME, Arbour L, Barrett I, Kalousek DK Prenatally detected trisomy 20 mosaicism. Prenat Diagn 2005;25:239-244.

13. Powis Z, Erickson RP. Uniparental disomy and the phenotype of mosaic trisomy 20: a new case and review of the literature. J App/ Genet 2009;50: 293-296.

14. Bastepe $\mathrm{M}$, Lane $\mathrm{AH}$, Jüppner H. Paternal uniparental isodisomy of chromosome $20 q$-and the resulting changes in GNAS1 methylation-as a plausible cause of pseudohypoparathyroidism. Am J Hum Genet 2001;68:1283-1289.

15. Long DN, McGuire S, Levine MA, Weinstein LS, Germain-Lee EL. Body mass index differences in pseudohypoparathyroidism type 1a versus pseudopseudohypoparathyroidism may implicate paternal imprinting of Galpha(s) in the development of human obesity. J Clin Endocrinol Metab 2007;92: 1073-1079.

16. Plagge A, Gordon E, Dean W, et al. The imprinted signaling protein XL alpha s is required for postnatal adaptation to feeding. Nat Genet 2004;36:818-826.

17. Xie T, Plagge A, Gavrilova O, et al. The alternative stimulatory G protein alphasubunit XLalphas is a critical regulator of energy and glucose metabolism and sympathetic nerve activity in adult mice. J Biol Chem 2006:281:18989-18999.

18. Yu S, Gavrilova O, Chen H, et al. Paternal versus maternal transmission of a stimulatory G-protein alpha subunit knockout produces opposite effects on energy metabolism. J Clin Invest 2000;105:615-623.

19. Allen EG, Freeman SB, Druschel $C$, et al. Maternal age and risk for trisomy 21 assessed by the origin of chromosome nondisjunction: a report from the Atlanta and National Down Syndrome Projects. Hum Genet 2009;125:41-52. 